\section{EFEITO DA INTERRUPÇĀO \\ TEMPORARIA DO ALEITAMENTO DE BEZERROS NA FERTILIDADE DE UM REBANHO DE VACAS NELORE}

\begin{abstract}
CARLOS GUILHERME DORILEO LEITE Pds-graduado Faculdade de Medicina Veterinaria e
\end{abstract} Zootecnia da USP

VALQUIRIA HYPPOLITO BARNABE Professor Adjunto Faculdade de Medicina Veterinaria e Zootecria da USP

JOSE ANTONIO VISINTIN

Professor Assistente Doutor Faculdade de Medicina Veterinaria e Zootecnia da USP

RENATO CAMPANARUT BARNABE Professor Titular Faculdade de Medicina Veterinaria e Zootecnia da USP

LEITE, C.G.D.; BARNABe, V.H.; VISINTIN, J.A.; BRRNABE, R.C. Ef eito da interrupço temporária do aleitamento de bezerros na fertilidade de um rebanho de vacas Nelore. Rev. Fac. Med. Vet. Zootec. Univ. 5. Paulo, 25(2): 243.249, 1988.

RESUMO: 0 experimento foi realizado no municipio de Pocone, Pantanal Matogrossense, Brasil, utilizando 75 vacas da raca Nelore, em criacgo extensiva, cujos bezerros estavam com 3 a 4 meses de idade. Os animais foram separados em 3 grupos de 25, identificados por brincos de cores azul, vermelha e amarela. Os grupos amarelo e vermelho sofreram interrupcăo do aleitamento por meio de tabuletas especiais fixadas nos focinhos dos bezerros, respectivamente, por 10 e 15 dias. 0 grupo azul figurou como testemunha. A ocorrência de cio foi observada diariamente durante 45 dias, nos horarios de 7 as 8 e das 17 as 18 horas. - diagnóstico de gestaço foi efetuado atraves de palpacaro retal, decorridos 60 dias do termino do experimento. A interrupcao do aleitamento, independentemente se realizada por 10 ou 15 dias, aumentou em 41\% o numero de vacas em cio e acarretou cerca de $40 \%$ a mais de vacas prenhes.

UNITERMOS: Desmame precoce; Fertilidade; Vacas nelore

\section{INTRODUCAO}

No Brasil, apesar das grandes areas de pastagens, do clima relativamente proplcio e do regime pluviometrico suficiente, a fertilidade do rebanho bovino de corte estámuito abaixo dos padrós exigidos pela moderna produtividade pecuária.

$$
\text { Por outro lado, a ovulacao e a }
$$
atividade reprodutiva a ela relacionada sa suprimidas por um variável periodo de tempo apds o parto e durante a lacta६ צ० (CLAPP, 2,1937 )

Periodos maic extensos de anestro so verificados em vacas em amamentaço, quando comparados com as de vacas secas (CASIDA et alii, 1, 1968; MIES FILHO et alii, 12, 1970), com os de animais ordenhados duas vezes ao dia (GRAVEs et alii, 4, 1968) ou com os daqueles que sofrem remoço do estimulo da mamada 24 horas apos o parto (OXENREIDER, 15, 1968)

Assim, varias técnicas envolvendo os bezerros têm sido preconizadas, com a finalidade de reduzir o perlodo de anestro pos parto em vacas de corte, dentre elas: o desmame precoce (SHORT et alii, 21, 1972; SMITH JR \& VICENT, 23, 1972; LASTER et alii, 8, 1973; PIMENTEL et alii, 16, 1979; NEUILLE \& MeCORMICK, 14, 1981); os efeitos da PGF 2 assaciada ou n̊o ao desmame temporário por sete dias (MACIEL \& MIES FILHO, 11, 1985); a interferencia na frequência da amamentaᄃ I o (RANDEL \& WELKER, 18, 1978; RANDEL, 17, 1981; LA VOIE et alii, 9 1981); tratamento hormonal das vacas mais a remoça do bezerro (HERRNSHAW, 6, 1978; MIES FILHO \& SA, 13, 1978); 5MITH et alii，22, 1979; TROXEL et alii, 24 , 1980; WALTERS et alii, 25, 1982; MACIEL, $10,1982)$ e a interrupça temporaria do aleitamento por curtos perlodos de 48 horas (WILTBANK, 26, 1974) ou entao, por prazos maiores, mediante dispositivos especiais colocados no focinho do bezerro (ROSA \& LERL, 30, 1976; RIBEIRO, 19, 1983).

Neste trabalho, visou-se verificar os efeitos da interrupca do aleitamento mediante a utilizaço de dispositivas (tabuletas) fixados na extremidade do focinho dos bezerras, sobre a fertilidade de um rebanho bovino de raca $\mathrm{Ne}$ lore, criado no municipio de Pocone, Estado do Mato Grosso.

MATERIAL E METODO 
Estado do Mato Grosso, durante o perlodo de 29 de setembro a 13 de novembro de 1984, utilizando-se 75 vacas de raca Nelore, com idades variando de 4 a 10 anos e com bezerros de 3 a 4 meses ao pé. As vacas foram separadas aleatoriamente em 3 lotes de 25 , identificadas por brincos plasticas numerados de 01 a 25 e de cores diferentes, assim distribuldos:

- azul - grupo controle com aleitamento livre.

- amarelo - grupu com aleitamento interrompido por $10 \mathrm{dias}$

- vermelho - grupo com aleitamento interrompido por 15 dias.

Para impedir a amamentaça foram utilizadas tabuletas de forma ovalada, * medindo $10 \mathrm{~cm}$ de altura por $15 \mathrm{~cm}$ de largura, pesando $50 \mathrm{~g}$. aproximadamente e fabricadas com foltha de propileno. Estas tabuletas foram fixadas no septo nasal dos bezerros por meio de dispositivo especial graduado com duas esferas nas extremidades, situadas em solucăo de continuidade retangular da borda do aparelhu. Enquanto a face posterior da tabuleta é lisa, a face anterior apresenta pontas agucadas, com cerca de $1 \mathrm{~cm}$ de comprimento e dirigidas para a frente, impedindo assim a amamentaço.

Todos os animais foram mantidos em sistema de rotaça de pastagens em três areas contlguas de apraximadamente 30 hectares cada uma, com relevo uniforme e predominância de Brachiaria humidicula e, ainda, suplementaça de sal mineral** jưntamente com sal comum nos cochos.

Devido a problemas de estiagem, as vacas foram mantidas separadas de touros durante 15 dias antes do inlcio do experimento, juntando-se, entao, três reprodutares da mesma raca, de seis anos de idade aproximadamente e cujos quadros espermaticos revelaram estar dentro dos padroes normais para reproducăo. Embora com utilizaço de cobertura natural, os

\section{* BOVITEC}

** SOROCABRNO

*** CHINBALL touros foram providos de bucal marcador***, observando-se as fêmeas por um mês e meio durante dois perlodos dia. rios, isto e, das 7 as 8 e das 17 as 18 horas, segundo sistema empregado em inseminaço artificial, com a finalidade de detectar o inlcio da atividade ovariana pos parto. O diagnostico de gestaço das vacas foi feito por palparyo retal, 60 dias apos o termino do experimento.

Ds resultados obtidos foram submetidos à analise de variância e ajuste de modelos lineares (GRIZZLE et alii, 5 , 1969). As atividades de organizaço e manipulaço do arquivo de dados experimentais e sua analise estatistica foram realizadas atraves do conjunto de subrotinas BMDP (DIXON, 3, 1981) e do programa GENCAT (LANDIS et alii, 7,1976 ), implantados no Centro de Computacá Eletranica da Universidade de 5 go Paulo.

\section{RESULTADOS E DISCUS5RO}

A atuaço do homem sobre o processo da amamentacăo dos bezerros, embora utilizando diferentes tecnicas, visou sempre o mesmo e unico objetivo de reduzir o anestro pos parto e, consequentemente, aumentar a fertilidade do rebanho, conforme comprovaram os trabalhos de SHORT et ali i, 21 (1972); SMITH JR \& VINCENT, 23 (1972); LASTER et alii, 8, (1973); WILTBANK, 24 (1974); HERRNSHAW 6 (1978); RANDEL \& WELKER, 18 (1978); PIMENTEL et alii, 16 (1979); 5MITH et alii, 22 (1979); TROXEL et alii, 24 (1980); LA VOIE et alii, 9 (1981); NEVILLE \& MACCORMICK, 14 (1981); RANDEL, 17 (1981); MACIEL, 10 (1982); WALTERS et ali 25 (1982)

As frequências observadas neste trabalho, bem como as proporçes de ocorrencia de cio e prenhez, estao indicadas respectivamente, nas Tab. 1 e 2 .

TABELA 1 - Frequência observada de cio em rebanho bovi no de raca Nelore submetido a aleitamento interrompido. Pocone, MT, 1984.

\begin{tabular}{|c|c|c|c|c|c|c|}
\hline CATEGOR I A & CONTROLE & $\begin{array}{l}\text { INT } \\
\text { POR }\end{array}$ & $\begin{array}{l}\text { RRUPCPO } \\
10 \text { DIAS }\end{array}$ & $\begin{array}{l}\text { INTE } \\
\text { POR }\end{array}$ & $\begin{array}{l}\text { RRUPCAO } \\
15 \text { DIAS }\end{array}$ & TOTAL \\
\hline & & & & & & \\
\hline & $(208)$ & 13 & $(52 x)$ & 17 & $(68 \%)$ & 35 \\
\hline CIO & $(80 x)$ & 12 & $(48 x)$ & 8 & $(32 x)$ & 40 \\
\hline TOTAL & $25(100 x)$ & 25 & $(1008)$ & 25 & $(1008)$ & 75 \\
\hline
\end{tabular}


Efeito da interrupçăo temporária do aleitamento de bezerros na fertilidade

Tabela 2 - Frequencia observada de prenhez em rebanho
bovino de raca Nelore submetido a aleitamen-
to interrompido. Pocone, MT, 1984.
to interrompida. Pocone, MT, 1984.

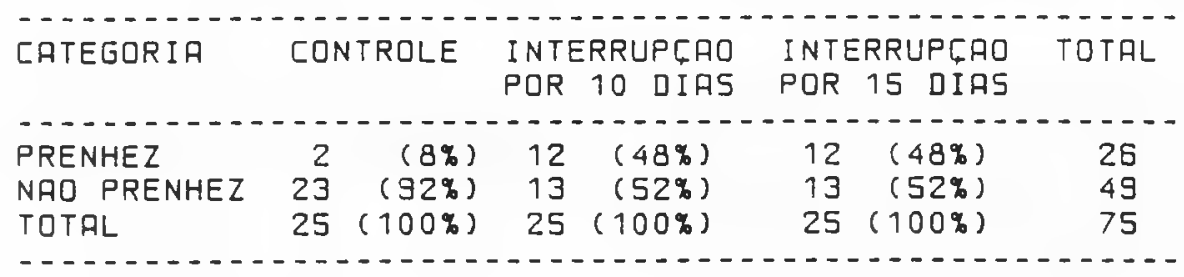

\begin{abstract}
A menor porcentagem de animais em cio do grupo controle caracteriza o anestro pos parto com supressão da ovulacăo e da atividade reprodutiva durante a Lactaça, conforme jà descreveu CLAPP, 2 (1937), corroborado por CASIDA et alii, 1 (1968) e MIES FILHO et alii, 12 (1970). Confirmando ainda esses resul. tados, GRAVES at alii, 4 (1968) e OXENREIDER, 15 (1968) relataram que é maior o anestro em vacas amamentando do que o daquelas ordenhadas duas vezes ao dia, ou mesmo o de outras com interrup̧a do estimulo da mamada 24
\end{abstract}

horas apos o parto. Nossos resultados indicaram que a interrupcăo do aleitamento, seja por 10 ou 15 dias, acarretou um aumento de, aproximadamente, $41 \%$ ( 11\%) no numero de vacas em cio, diferenca esta altamente significativa $(P=0,0003)$ en relaça ao grupo controle. MIES FILHO \& SA, 13 (1978) verificaram que a ocorrência de cio aos quatro dias de observacao, foi de $10 \%$ para o desmame de 84 horas.

A Tab. 3 confirma os dados da Tab. 1, estabelecendo comparacoes dos grupos experimentais 2 a 2 das proporfaes de ocorrência de cio.

TABELA 3 - Estatistica de comparacós das proporcores de ocorrência de cio atraves da tecnica de Grizzle, Starmer e Koch (1969) em rebanho bovino de raca Nelore submetido a aleitamento interrompido. Pocone, MT, 1984.

\begin{tabular}{|c|c|c|c|}
\hline COMPARACOES & ESTAT I ST ILA & $\begin{array}{l}\text { GRAUS DE } \\
\text { LIBERDADE }\end{array}$ & $\begin{array}{c}\text { NIVEL DESCRITIVO } \\
\text { (p-value) }\end{array}$ \\
\hline $\begin{array}{l}\text { Homogeneidade dos } \\
\text { três grupos }\end{array}$ & 16,2488 & 2 & $\begin{array}{c}0,0003 \\
0, \ldots\end{array}$ \\
\hline $\begin{array}{l}\text { Igualdade dos grupos- } \\
\text { controle e interrupcajo } \\
\text { por } 10 \text { dias }\end{array}$ & 6,2500 & 1 & 0,0124 \\
\hline $\begin{array}{l}\text { Igualdade dos grupas- } \\
\text { contrale e interrupcago } \\
\text { por } 15 \text { dias }\end{array}$ & 15,2542 & ------ & 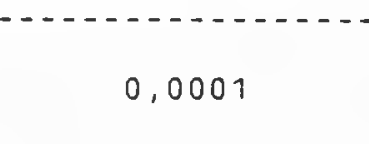 \\
\hline $\begin{array}{l}\text { Igualdade dos grupos- } \\
\text { controle e interrupcago } \\
\text { por } 10 \times 15 \text { dias }\end{array}$ & 1,3699 & ----- & - - - - - \\
\hline
\end{tabular}

A Tab. 4, confirmando os dados da Tab. 2, demonstra uma diferenca altamente significativa entre os grupos experimentais, relativamente a proporqa de ocorrencia de prenhez ( $p=0,000)$ e contém, também, resultados referentes as comparaçes dos grupos 2 a 2 . Verificouse, portanto, que a interrupcro do aleitamento acarretou aumento do numero de vacas prenhes de, aproximadamente, $40 \%$ ( \pm 9\%), nao importando se a interrupcao foi de 10 ou 15 dias. 
TABELA 4 - Estatistica das comparaçes das proporcoses de acorrência de prenhez atraves da tecnica de Grizzle, Starmer e Koch (1969) em rebanho bovino de raca Nelore submetido a aleitamento interrompido. Pocone, MT. 1984 .

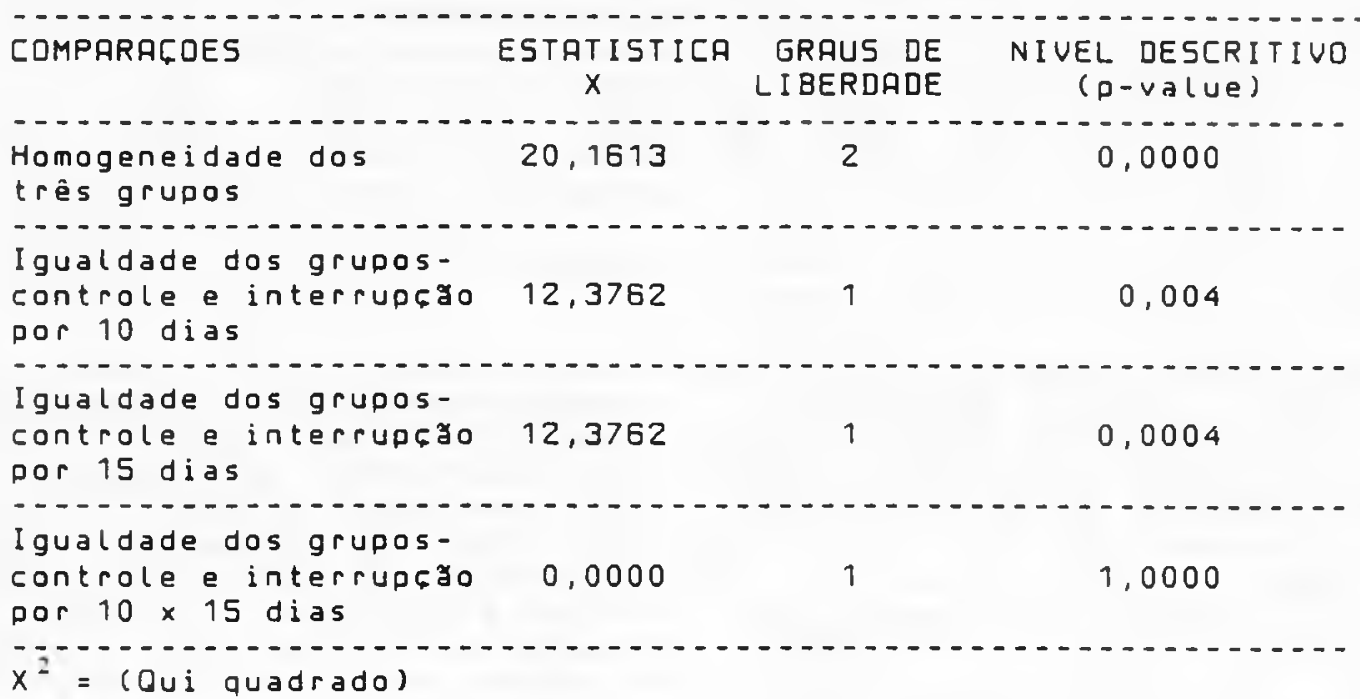

Ambos os grupos submetidos à interrupcgo do aleitamento, seja por 10 ou 15 dias, apresentaram igual numero de vacas prenhes ( 12 em 25 animais), o que equivale a uma taxa de concepcaro de $48 \%$. Este resultado é ligeiramente superior ao apresentado por RIBEIRO, 19, (1983), com utilizaço de tabuletas por 7 dias, porém inferior àqueles relatados por ROSA \& LERL, 20 (1976). Estes autores, mediante a utilizaça de tabuletas por 7 dias, obtiveram $70 \%$ de prenhez e conseguiram resultados ainda melhores ( $86 \%$ ) quando o dispositivo nasal impediu os bezerros de mamar por 13 dias. MACIEL \& MIES FILHO, 11 (1985) tambem conclulram que o desmame temporario revelou-se pratica de manejo eficaz a partir do 30 dia de sua adocaro, melhorando significa- tivamente a fertilidade, tanto dos cios espontâneos, como dos induzidos pela $\operatorname{PCF}_{2} \alpha$ No caso presente, tendo em vista que nao foi detectada diferenca significativa entre os grupos com interrupcaro do aleitamento por 10 e 15 dias ( $p=$ 0,2418 e $p=\{, 000)$, respectivamente, os animais correspondentes foram reagrupados com a finalidade de melhorar a precisao da estimativa de diferenca nas proporcốs de ocorrência de cio e prenhez. Os resultados correspondentes à reanalise estao indicados na Tab. 5 , confirmando os fatos já anteriormente discutidos, mostrando que o $x^{2}$ em relaço à prenhez é maior do que $x^{2}$ em relaço ao cio, indicando isto uma possivel maior interferência do aleitamento interrompido em relaça à prenhez.

TABELA 5 - Efeito da interrupcaro do aleitamento na proporca de ocorrencia de cio e prenhez, atraves da tecnica de Grizzle, Stamer e Koch (1969), em rebanho bovino de raca Nelore. Pocone, MT. 1984.

\begin{tabular}{|c|c|c|c|}
\hline COMPARACOES & ESTAT I $x^{2}$ ICA & $\begin{array}{l}\text { GRAUS DE } \\
\text { LIBERDADE }\end{array}$ & $\begin{array}{c}\text { NIVEL DESCRI.T IVO } \\
\text { ( } p-v a \text { lue })\end{array}$ \\
\hline $\begin{array}{l}\text { Efeito da interrupcăo } \\
\text { do aleitamento na } \\
\text { ocorréncia de cio }\end{array}$ & 14,8789 & 1 & 0,0001 \\
\hline $\begin{array}{l}\text { Efeito da interrupcao } \\
\text { do aleitamento na } \\
\text { ocorrencia de prenhez }\end{array}$ & 20,1613 & 1 & 0,0000 \\
\hline
\end{tabular}

Rev. Fac. Med. Vet. Zootec. Univ. S. Paulo, 25(2):243-249, 1988. 
CONCLUSOES

Os resultados obtidos no presente experimento permitiram extrair as seguintes conclusobs:

a interrupca do aleitamento acarretou um aumento de, aproximadamente,

a - 41\% ( $\pm 11 \%)$ de vacas em cio

b - 40\% ( $\pm 9 \%$ ) de vacas prenhez, na importando se essa interrupcra foi de dez ou quinze dias.

2 - em funça dos valores obtidos quando da analise do $x^{2}$ (quiquadrado), referente à influência do aleitamento sobre o aparecimento de cio e - estabelecimento de prentiez, parece llaito dizer que foi maior sobre o segundo evento.
LEITE, C.G.D.; BARNABE, U.H.; UISINTIN, J.A.; BRRNABE, R.C. Effect of interrupted weaning on fertility in a herd of Nelore cows. Rev. Fac. Med. Vet. Zootec. Univ. 5. Paulo, 25(2): 243-249, 1988.

SUMMARY: This experiment was conducted at Pocone county in the Pantanal of Mato Grosso 5tate, Brazil, including 75 Nelore cows under range conditions with their calves 3 to 4 months of age. Cows were separated into 3 groups of 25 , each group with ear tags of different colours: blue, red and yellow. Calves of red and vellow groups were subjected to interrupted weaning for 10 and 15 days, respectively, through a device in their noses. Blue group acted as control. Estrus was detected by twice a day observations during 45 days, from 7 to 8 a.m. and 5 to $6 \mathrm{p} . \mathrm{m}$. Pregnancy diagnosis was made through rectal palpation 60 days after the end of the experiment. Interrupted weaning, for 10 or 15 days, increased both number of cows in estrus ( $47 \%$ ) and pregnancy rate $(40 \%)$.

UNITERMS: Weaning; Fertility; Nelore cows

\section{REFERENCIAS BIBLIOGRAFICRS}

1 - CASIDA, L.E.; GRAVES, W.E.; HAUSER, E.R.; LAUDERDALE, J.W.; RIESEN, H.W.; SAIDUDDIN, S.; TYLER, W.J. Studies on the pospartum cow: review of literature. Bull. Wis. agric. Exp. Stn., 270:1-54, 1968 .

2 - CLAPP, H. A factor in breeding efficiency in cattle. Rec. Proc. Amer.. Soc. Anim. Prod., $30: 259-265,1937$. sof tware. Berkeley, University of California Press, 1981.
4 - GRAVES, W.E.; LAUDERDALE, J.W.; HAUSER, E.R.; CASIDA, L.E. Relation of pospartum interval to pituitary gonadotropin. ovarian follicular development and fertility in beef cows. Bull. Wis. agric. Exp. Stn. $270: 23-26$, 1968 .

5 - GRIZZLE, J.E.; STAMER, C.F.; COOK, G.G. Analysis of categorical data by linear models. Biometrics, 25:489-504, 1969.

6 - HERRNSHAW, H. Effect of temporary calf removal on oestrus activity 
248 LEITE. C.G.D. et alii

Efeito da interrupça temporária do aleitamento de bezerros na fertilidade

in cows. Proc. Aust. Soc. Anim. Prod., 12:258, 1978 .

7 - LANDIS, J.R.; STANISH, W.M.; FREEMAN, J.L.; KOCH, G.G. A computer program for the generalized chi-square analysis of categorial data using weight least squares (GENCAT). Comput. Progr. Biomed., 6:196-231, 1376.

8 - LASTER, D.B.; GLIMP, H.A.; GREGORY, K.E. Effects of early weaning on pospartum reproductive activity and uterine involution in beef cows. J. anim. 5ci., $36: 734-740,1973$.

S - LA VOIE, U.; HAN, D.L.; FOSTER, D.B.; MOODY, E.L. Suekling effect on estrus and blood plasma progesterone in pospartum beef cows. J. anim. 5ci., 52:802-812, 1981 .

10 - MACIEL, A.5. Utilizaça da PGF,a associada ao desmame temporario em vacas de corte em amamentaఢ \%०. In: CONGRESSO BRASILEIRO DE MEDICINA VETERINARIA, 18. Camborid, 1982. Anais. p.177.

11 - MACIEL, A.S. \& MIES FILHO, A. Utilizacgo da PGF associada ao desmame temporario em vacas de corte em amamentaço. Rev. bras. Reprod. anim., 9:171-180, 1985 .

12 - MIES FILHO, A.; PRADO, O.T.G.; GAREIA, A.J.F.; FAGUNDES, A.U.; LOPEZ, J.L.M. ; SILUA, R.G. Inseminacao artificial no gado de corte no Rio Grande do Sul. In: CONGRESSO BRASILEIRO DE MEDICINA VETERINARIA, 12. Porto Alegre, 1970. Anais. p.349-361.

13 - MIES FILHO, A. \& SA, N.F. Sincronizaço do ciclo estral em vacas de corte em lactaço. Rev. bras. Reprod. anim, 2:21-24, 1978 .

14 - NEVILLE, W.E. \& MECORMICK, W.C. Performance of early and normal weaned beef calves and their dams. J. anim. Sci., 52:715$724,1981$.

15 - DXENREIDER, S.L. Effects of suckling and ovarian function on pospartum reproductive activity in beef cows. Amer. J. vet. Res., 29:2099-2102, 1968 .

16 - PIMENTEL, [.A.; DESCHAMPS, J.C.; OLIVEIRA, J.A.; PIMENTEL, M.A. Effects of early weaning on reproductive efficiency in beef cows. Theriogenology, 11:421427,1979

17 - RANDEL, R.D. Effect of once-daily suckling on pospartum interval and cow-calf performance of first calf Branman $x$ Hereford heifers. J. anim. 5ci., 53:755757, 1981

18 - RANDEL, R.D. \& WELKER, G.A. Effect of age at first calving and once daily suckling upon days open and calving interval. J. anim. Sci., 47:384, 1978 .

19 - RIBEIRD, W.N. Efeitos do zeranol e desmame interrompido sobre a eficiência reprodutiva em bovinos de corte. Pelotas, 1983. [Dissertaça de mestrado - Universidade Federal de Pelotas ]

20 - ROSA, N. \& RERL, C.M. Desmame interrompido. Nota previa. Novo metodo para aumentar a fertilidade do rebanho bovino. Aro. Fac. Vet. Univ. Fed. R.G. Sul, $4 / 5: 74-77,1976$

21 - SHORT, R.E.; BELLOWS, R.A.; MOODY, E.L.; HOWLAND, B.E. Effects of suckling and mastectomy on bovine pospartum reproduction. J. anim. 5ci., 34:70-74, 1972 .

22 - SMITH, M.F.; BURRELL, W.C.; SHIPP, L.D.; SPROTT, L.R.; SONGSTER, L.R.; SONGSTER, W.N.; WILTBANK, J.N. Hormone treatments and use of calf removal in pospartum beef cows. J. anim. Sci. $48: 1285-1294,1979$.

23 - SMITH JR., L.E. \& VICENT, C.K. Effects of early weaning and exogenous hormone treatment on bovine pospartum reproduction. J. anim. Sci., 35:1228-1232, 1972.

24 - TROXEL, T.R.; KESLER, D.J.; NOVLE, R.C.; CARLIN, S.E. Dvulation and reproductive hormones 
Efeito da interrupção temporária do aleitamento de bezerros na fertilidade

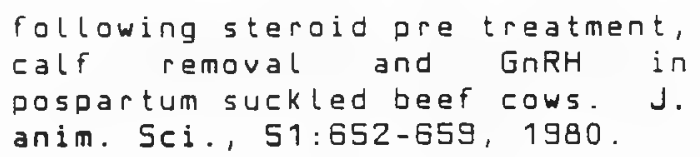

25 - WRLTERS, Q.L.; SMITH, M.F.; HARMS, P.G.; WILTBANK, J.N. Effect of steroids and or 48 hr. calf removal on serum luteinizing

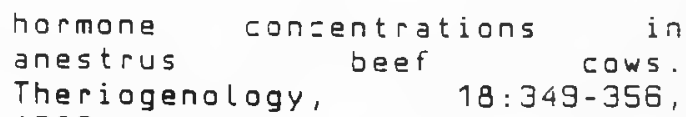

26

WILTBANK, J.N. Management programs to increase reproductive efficiency of beef herd. J. anim. Sci., 38:58-70, 1974.

Recebido para publicaca en 25/02/87 Aprovado para publicacao en $02 / 10 / 87$ 